\title{
LMI-Based Approach for Exponential Robust Stability of High-Order Hopfield Neural Networks with Time-Varying Delays
}

\author{
Yangfan Wang ${ }^{1}$ and Linshan Wang ${ }^{2,3}$ \\ ${ }^{1}$ College of Marine Life Science, Ocean University of China, Qingdao 266071, China \\ ${ }^{2}$ Department of Mathematics, Ocean University of China, Qingdao 266071, China \\ ${ }^{3}$ Department of Mathematics, Liaocheng University, Liaocheng 252059, China \\ Correspondence should be addressed to Linshan Wang, wenxuetzz@gmail.com
}

Received 30 January 2012; Accepted 27 March 2012

Academic Editor: Wan-Tong Li

Copyright (c) 2012 Y. Wang and L. Wang. This is an open access article distributed under the Creative Commons Attribution License, which permits unrestricted use, distribution, and reproduction in any medium, provided the original work is properly cited.

This paper studies the problems of global exponential robust stability of high-order hopfield neural networks with time-varying delays. By employing a new Lyapunov-Krasovskii functional and linear matrix inequality, some criteria of global exponential robust stability for the high-order neural networks are established, which are easily verifiable and have a wider adaptive.

\section{Introduction}

Hopfield neural networks (HNNs) with time delays and their various generalization have been successfully employed in many areas such as pattern recognition, associate memory, and combinatorial optimization (see [1-11]). Recently, the dynamics of high-order Hopfield neural networks (HOHNNs) with time delays have been considerable attention (see [12-18]), due to the neural networks have stronger approximation properties, faster convergence rate, greater storage capacity, and higher fault tolerance than lower order neural networks (see [12]). So, the stability of HOHNNs with time delays should be a focused topic of theoretical as wells as practical importance. This paper studies the problems of global exponential robust stability of high-order hopfield neural networks with time-varying delays. This paper is also an extension of our previous work [19]. By employing a new Lyapunov-Krasovskii functional and linear matrix inequality, some criteria of global exponential robust stability for the highorder neural networks are established, which are easily verifiable and have a wider adaptive. 


\section{Model Description and Preliminaries}

We consider the following HOHNNs with time-varying delays:

$$
\begin{aligned}
\frac{d u_{i}(t)}{d t}= & -a_{i} u_{i}(t)+\sum_{j=1}^{n} W_{i j} f_{j}\left(u_{j}(t)\right)+\sum_{j=1}^{n} T_{i j} g_{j}\left(u_{j}(t-\tau(t))\right) \\
& +\sum_{j=1}^{n} \sum_{l=1}^{n} T_{i j l} g_{j}\left(u_{j}(t-\tau(t))\right) g_{l}\left(u_{l}(t-\tau(t))\right)+V_{i}, \\
u_{i}\left(t_{0}+\theta\right)= & \xi_{i}(\theta), \quad-\tau_{0} \leq \theta \leq 0, i=1, \ldots, n, \\
& 0 \leq \tau(t) \leq \tau_{0}, \quad \underline{a}_{i} \leq a_{i}, \quad \underline{W}_{i j} \leq W_{i j} \leq \bar{W}_{i j}, \quad \underline{T}_{i j} \leq T_{i j} \leq \bar{T}_{i j}, \\
& \underline{T}_{i j l} \leq T_{i j l} \leq \bar{T}_{i j l}, \quad \underline{V}_{i} \leq V_{i} \leq \bar{V}_{i}, \quad i, j, l=1, \ldots, n,
\end{aligned}
$$

where $u(t)=\left(u_{1}(t), \ldots, u_{n}(t)\right)^{T} \in R^{n}, a_{i}>0, W_{i j}, f_{j}, g_{j}, V_{i}$ have the same meanings as those in [13], $T_{i j}, T_{i j l}$ are the first- and second-order synaptic weights of the system (2.1) (see [12]).

In this paper, the superscript " $T$ " presents the transpose.

We assume throughout that the neuron activation functions $f_{j}\left(u_{j}\right), g_{j}\left(u_{j}\right), j=1, \ldots, n$, satisfy the following conditions:

$$
\begin{gathered}
\left(H_{1}\right):\left|g_{j}\left(u_{j}\right)\right| \leq M_{j}, \quad 0 \leq \frac{\left|f_{j}\left(u_{j}\right)-f_{j}\left(v_{j}\right)\right|}{\left|u_{j}-v_{j}\right|} \leq l_{0 j}, \quad 0 \leq \frac{\left|g_{j}\left(u_{j}\right)-g_{j}\left(v_{j}\right)\right|}{\left|u_{j}-v_{j}\right|} \leq l_{1 j}, \\
\forall u_{j} \neq v_{j}, u_{j}, v_{j}, l_{0 j}, l_{1 j} \in R .
\end{gathered}
$$

From (2.2), we know

$$
\begin{gathered}
\left|f\left(u^{1}\right)-f\left(u^{2}\right)\right| \leq\left|L_{0}\left(u^{1}-u^{2}\right)\right|, \\
\left|g\left(u^{1}\right)-g\left(u^{2}\right)\right| \leq\left|L_{1}\left(u^{1}-u^{2}\right)\right|, \quad \forall u^{1}, u^{2} \in R^{n},
\end{gathered}
$$

where $u^{i}=\left[u_{i 1}, \ldots, u_{i n}\right]^{T} \in R^{n}, f\left(u^{i}\right)=\left[f_{1}\left(u_{i 1}\right), f_{2}\left(u_{i 2}\right), \ldots, f_{n}\left(u_{i n}\right)\right]^{T}, g\left(u^{i}\right)=$ $\left[g_{1}\left(u_{i 1}\right), g_{2}\left(u_{i 2}\right), \ldots, g_{n}\left(u_{i n}\right)\right]^{T}, i=1,2 . L_{0}=\operatorname{diag}\left(l_{01}, l_{02}, \ldots, l_{0 n}\right)$, and $L_{1}=\operatorname{diag}\left(l_{11}, l_{12}, \ldots, l_{1 n}\right)$.

If there is an equilibrium point $u^{*}=\left[u_{1}^{*}, \ldots, u_{n}^{*}\right]^{T}$ of system $(2.1)$, we can rewrite system (2.1) as the following equivalent form:

$$
\begin{aligned}
\frac{d\left(u_{i}(t)-u_{i}^{*}\right)}{d t}= & -a_{i}\left(u_{i}(t)-u_{i}^{*}\right)+\sum_{j=1}^{n} W_{i j}\left(f_{j}\left(u_{j}(t)\right)-f_{j}\left(u_{j}^{*}\right)\right) \\
& +\sum_{j=1}^{n}\left(T_{i j}+\sum_{l=1}^{n}\left(T_{i j l}+T_{i l j}\right) \zeta_{l}\right)\left(g_{j}\left(u_{j}(t-\tau(t))\right)-g_{j}\left(u_{j}^{*}\right)\right)
\end{aligned}
$$


Journal of Applied Mathematics

$$
\begin{aligned}
u_{i}\left(t_{0}+\theta\right)= & \xi_{i}(\theta), \quad-\tau_{0} \leq \theta \leq 0, i=1, \ldots, n . \\
& 0 \leq \tau(t) \leq \tau_{0}, \quad \underline{a}_{i} \leq a_{i}, \quad \underline{W}_{i j} \leq W_{i j} \leq \bar{W}_{i j}, \quad \underline{T}_{i j} \leq T_{i j} \leq \bar{T}_{i j}, \\
& \underline{T}_{i j l} \leq T_{i j l} \leq \bar{T}_{i j l}, \quad i, j, l=1, \ldots, n,
\end{aligned}
$$

where $\zeta_{l}=(1 / 2)\left(g_{l}\left(u_{l}(t-\tau(t))\right)+g_{l}\left(u_{l}^{*}\right)\right)$ and $\left|\zeta_{l}\right| \leq M_{l}$.

We easily obtain that system (2.4) is equivalent to system (2.1) (see [13]).

The notations in this paper are quite standard:

(i)

$$
\|u(t)\|=\left(\sum_{i=1}^{n}\left|u_{i}(t)\right|^{2}\right)^{1 / 2}
$$

where $\left|u_{i}(t)\right|$ denotes Euclid's norm.

(ii) $A=\left(a_{i j}\right)_{n \times n}>0(<0)$ : a positive (negative) definite matrix, that is, $x^{T} A x>0(<0)$ for any $x \in R^{n}$.

(iii) $A=\left(a_{i j}\right)_{n \times n} \geq 0$ : a semipositive definite matrix, that is, $x^{T} A x \geq 0$ for any $x \in R^{n}$.

(iv) $A \geq B$ (resp., $A>B$ ): this means $A-B$ is a semi-positive definite matrix (resp., positive definite).

(v) $I$ : identity matrix with compatible dimension.

(vi) $I^{\circ}=(1,1, \ldots, 1)^{T}$.

(vii) $\lambda_{\max }(A)$ (resp., $\left.\lambda_{\min }(A)\right)$ means the largest (resp., smallest) eigenvalue of the matrix A.

(viii) $C\left(\left[-\tau_{0}, 0\right], R^{n}\right)$ denotes a set of continuous functions.

Let $u(t, \xi)=\left[u_{1}(t, \xi), \ldots, u_{n}(t, \xi)\right]^{T}$ denote the solution $u(t, \xi)$ to system (2.1) from the initial data $u\left(t_{0}+\theta, \xi\right)=\xi(\theta)$ on $-\tau_{0} \leq \theta \leq 0$ in $\xi(\theta) \in C\left(\left[-\tau_{0}, 0\right], R^{n}\right)$.

Definition 2.1. The equilibrium point $u^{*}$ of system (2.1) is called globally exponentially robustly stable on norm $\|\cdot\|$, if for any $\xi(\theta) \in C\left(\left[-\tau_{0}, 0\right], R^{n}\right)$ there exist scalars, $J>0$ and $\alpha>0$ such that the solution $u(t, \xi)$ to system (2.1) with the initial condition $u\left(t_{0}+\theta, \xi\right)=\xi(\theta)$ on $-\tau_{0} \leq \theta \leq 0$ satisfies

$$
\left\|u(t, \xi)-u^{*}\right\|^{2} \leq J e^{-\alpha t} \sup _{-\tau_{0} \leq \theta \leq 0}\left\|\xi(\theta)-u^{*}\right\|^{2}
$$

Lemma 2.2 (see [20]). The LMI

$$
\left[\begin{array}{ll}
Q(t) & S(t) \\
S^{T}(t) & R(t)
\end{array}\right]>0
$$


where $Q(t)=Q^{T}(t), R(t)=R^{T}(t)$, and $S(t)$ depend on $t$, is equivalent to any one of the following conditions:

$$
\begin{array}{ll}
\left(K_{1}\right), R(t)>0, & Q(t)-S(t) R^{-1}(t) S^{T}(t)>0 \\
\left(K_{2}\right), Q(t)>0, & R(t)-S^{T}(t) Q^{-1}(t) S(t)>0 .
\end{array}
$$

Lemma 2.3 (see [20]). Let $x \in R^{n}, y \in R^{n}$, and $\varepsilon>0$. Then, one has

$$
x^{T} y+y^{T} x \leq \varepsilon x^{T} x+\varepsilon^{-1} y^{T} y .
$$

\section{Main Results}

Let

$$
\begin{gathered}
M=\left[M_{1}, M_{2}, \ldots, M_{n}\right]^{T}, \quad \Gamma=\operatorname{diag}(M, M, \ldots, M), \quad \zeta=\left[\zeta_{1}, \zeta_{2}, \ldots, \zeta_{n}\right]^{T}, \\
\Pi=\operatorname{diag}(\zeta, \zeta, \ldots, \zeta), \quad \underline{A}=\operatorname{diag}\left(\underline{a}_{i}\right), \quad W=\left(W_{i j}\right)_{n \times n^{\prime}} \quad W^{+}=\left(W_{i j}\right)_{n \times n^{\prime}} \\
\bar{W}=\left(\max \left\{\left|\underline{W}_{i j}\right|,\left|\bar{W}_{i j}\right|\right\}\right)_{n \times n^{\prime}} \quad \bar{W}^{T}=\left(\max \left\{\left|\underline{W}_{j i}\right|,\left|\bar{W}_{j i}\right|\right\}\right)_{n \times n^{\prime}} \\
T=\left(T_{i j}\right)_{n \times n^{\prime}} \quad T^{+}=\left(T_{i j}\right)_{n \times n^{\prime}} \\
\bar{T}=\left(\max \left\{\left|\underline{T}_{i j}\right|,\left|\bar{T}_{i j}\right|\right\}\right)_{n \times n^{\prime}} \quad \bar{T}^{T}=\left(\max \left\{\left|\underline{T}_{j i}\right|,\left|\bar{T}_{j i}\right|\right\}\right)_{n \times n^{\prime}} \\
T_{i}=\left(T_{i j l}\right)_{n \times n^{\prime}} \quad T_{i}^{T}=\left(T_{i l j}\right)_{n \times n^{\prime}} \quad T_{i}^{+}=\left(T_{i j l}\right)_{n \times n^{\prime}} \\
\bar{T}_{i}=\left(\max \left\{\left|\underline{T}_{i j l}\right|,\left|\bar{T}_{i j l}\right|\right\}\right)_{n \times n^{\prime}} \quad \bar{T}_{i}^{T}=\left(\max \left\{\left|\underline{T}_{i l j}\right|,\left|\bar{T}_{i l j}\right|\right\}\right)_{n \times n} \\
T_{H}=\left(T_{1}+T_{1}^{T}, T_{2}+T_{2}^{T}, \ldots, T_{n}+T_{n}^{T}\right)_{n^{2} \times n^{\prime}}^{T} \\
T_{H}^{+}=\left(T_{1}^{+}+\left(T_{1}^{T}\right)^{+}, T_{2}^{+}+\left(T_{2}^{T}\right)^{+}, \ldots, T_{n}^{+}+\left(T_{n}^{T}\right)^{+}\right)_{n^{2} \times n^{\prime}}^{T} \\
\bar{T}_{H}=\left(\bar{T}_{1}+\bar{T}_{1}^{T}, \bar{T}_{2}+\bar{T}_{2}^{T}, \ldots, \bar{T}_{n}+\bar{T}_{n}^{T}\right)_{n^{2} \times n^{\prime}}^{T} \\
W_{\Pi}=T+\Pi^{T} T_{H}, \quad \bar{W}_{\Pi}=\bar{T}+\Pi^{T} \bar{T}_{H} .
\end{gathered}
$$

For the purpose of simplicity, we rewrite the system (2.4) as the following vector form:

$$
\begin{aligned}
\frac{d y}{d t}= & -A y(t)+W F_{0}(y(t))+\left(T+\Pi^{T} T_{H}\right) F_{1}(y(t-\tau(t))) \\
y\left(t_{0}+\theta\right)= & \varphi(\theta), \quad-\tau_{0} \leq \theta \leq 0,0 \leq \tau(t) \leq \tau_{0}, \\
& \underline{a}_{i} \leq a_{i}, \quad \underline{W}_{i j} \leq W_{i j} \leq \bar{W}_{i j}, \quad \underline{T}_{i j} \leq T_{i j} \leq \bar{T}_{i j}, \\
& \underline{T}_{i j l} \leq T_{i j l} \leq \bar{T}_{i j l}, \quad i, j, l=1, \ldots, n,
\end{aligned}
$$


where $y=y(t)=u(t, \xi(\theta))-u^{*}$ for any $\xi(\theta) \in C\left(\left[-\tau_{0}, 0\right], R^{n}\right), F_{0}(y(t))=f(y(t)+$ $\left.u^{*}\right)-f\left(u^{*}\right), f\left(y(t)+u^{*}\right)=\left[f_{1}\left(y_{1}(t)+u_{1}^{*}\right), f_{2}\left(y_{2}(t)+u_{2}^{*}\right), \ldots, f_{n}\left(y_{n}(t)+u_{n}^{*}\right)\right]^{T}, F_{1}(y(t-\tau(t)))=$ $g\left(y(t-\tau(t))+u^{*}\right)-g\left(u^{*}\right), g\left(y(t-\tau(t))+u^{*}\right)=\left[g_{1}\left(y_{1}(t-\tau(t))+u_{1}^{*}\right), g_{2}\left(y_{2}(t-\tau(t))+\right.\right.$ $\left.\left.u_{2}^{*}\right), \ldots, g_{n}\left(y_{n}(t-\tau(t))+u_{n}^{*}\right)\right]^{T}, \varphi(\theta)=\xi(\theta)-u^{*}, \xi(\theta)=\left(\xi_{1}(\theta), \ldots, \xi_{n}(\theta)\right)^{T}$.

Theorem 3.1. Given a positive definite matrix $Q=L_{1}^{T} L_{1}>0$. The equilibrium of system (2.1) is globally exponentially robustly stable on norm $\|\cdot\|$ for any $\tau(t)$ satisfying $\dot{\tau}(t) \leq \eta<1$, if system (2.1) satisfies $\left(H_{1}\right)$ and

$$
\left(H_{2}\right): C=A-W^{+} L_{0}-W_{\Gamma}^{+} L_{1}
$$

is M-matrix,

where $W_{\Gamma}^{+}=T^{+}+\Gamma^{T} T_{H^{\prime}}^{+}$and

$$
\left(H_{3}\right):\left[\begin{array}{cccc}
\Lambda & L_{0} & \beta \bar{W}^{T} & \beta \bar{W}_{\Pi}^{T} \\
L_{0}^{T} & -I & 0 & 0 \\
\beta \bar{W} & 0 & -I & 0 \\
\beta \bar{W}_{\Pi} & 0 & 0 & -(1-\eta) I
\end{array}\right] \leq 0
$$

where $\bar{W}_{\Pi}=\bar{T}+\Pi^{T} \bar{T}_{H}$, and $\Lambda=\beta(\alpha I-2 \underline{A})+e^{\alpha \tau_{0}} Q, \beta>0$.

Proof of Theorem 3.1. Let

$$
\begin{aligned}
h_{i}\left(u_{i}, V_{i}\right)= & a_{i} u_{i}-\sum_{j=1}^{n} W_{i j} f_{j}\left(u_{j}\right)-\sum_{j=1}^{n} T_{i j} g_{j}\left(u_{j}\right) \\
& -\sum_{j=1}^{n} \sum_{l=1}^{n} T_{i j l} g_{j}\left(u_{j}\right) g_{l}\left(u_{l}\right)+V_{i}=0, \quad i=1, \ldots, n .
\end{aligned}
$$

It is obvious that the solutions to (3.5) are the equilibrium points of system (2.1).

Let us define homotopic mapping as follows:

$$
H(u, \lambda)=\left(H_{1}\left(u_{1}, \lambda\right), \ldots, H_{n}\left(u_{n}, \lambda\right)\right)^{T},
$$

where

$$
H_{i}\left(u_{i}, \lambda\right)=\lambda h_{i}\left(u_{i}, V_{i}\right)+(1-\lambda) u_{i}, \quad \lambda \in[0,1]
$$

By homotopy invariance theorem (see, [21]), topological degree theory (see, [22]), $\left(H_{1}\right)-\left(H_{2}\right)$, and the proof which is similar to Theorem 3.1 in [23], we can conclude that (3.5) has at least one solution. That is, system (2.1) has at least an equilibrium point. 


\subsection{Part 2- Globally Exponentially Stable}

Define a Lyapunov-Krasovskii functional candidate by

$$
V(y(t))=\beta e^{\alpha t} y^{T}(t) y(t)+e^{\alpha \tau_{0}} \int_{t-\tau(t)}^{t} e^{\alpha \theta} y^{T}(\theta) Q y(\theta) d \theta>0
$$

where $Q=Q^{T}>0$.

From system (3.2), its Dini derivative can be calculated as

$$
\begin{aligned}
D^{+} V(y(t))=e^{\alpha t}[ & \alpha \beta y^{T}(t) y(t)+y^{T}(t)\left(-\beta A^{T}-\beta A\right) y(t) \\
& +\beta\left(F_{0}^{T}(y(t)) W^{T} y(t)+y^{T}(t) W F_{0}(y(t))\right) \\
& +\beta F_{1}^{T}(y(t-\tau(t)))\left(T+\Pi^{T} T_{H}\right)^{T} y(t) \\
& \left.+y^{T}(t)\left(T+\Pi^{T} T_{H}\right) F_{1}(y(t-\tau(t)))\right] \\
+ & e^{\alpha \tau_{0}}\left[e^{\alpha t} y^{T}(t) Q y(t)-e^{\alpha(t-\tau(t))} y^{T}(t-\tau(t)) Q y(t-\tau(t))(1-\dot{\tau}(t))\right]
\end{aligned}
$$

Since $0 \leq \tau(t) \leq \tau_{0}, \dot{\tau}(t) \leq \eta<1$, and $Q=L_{1}^{T} L_{1}>0$, we have

$-e^{\alpha \tau_{0}} e^{\alpha(t-\tau(t))} y^{T}(t-\tau(t)) Q y(t-\tau(t))(1-\dot{\tau}(t)) \leq-e^{\alpha t} y^{T}(t-\tau(t)) Q y(t-\tau(t))(1-\eta)$,

From Lemma 2.3 and (2.3), we have

$$
\begin{aligned}
& e^{\alpha t} \beta\left(F_{0}^{T}(y(t)) W^{T} y(t)+y^{T}(t) W F_{0}(y(t))\right) \\
& \leq e^{\alpha t} \beta\left(\beta y^{T}(t) W W^{T} y(t)+\frac{1}{\beta} F_{0}^{T}(y(t)) F_{0}(y(t))\right) \\
& \leq e^{\alpha t} y^{T}(t)\left(\beta^{2} W W^{T}+L_{0}^{T} L_{0}\right) y(t) \leq e^{\alpha t} y^{T}(t)\left(\beta^{2} \bar{W} \bar{W}^{T}+L_{0}^{T} L_{0}\right) y(t), \\
& e^{\alpha t} \beta\left(F_{1}^{T}(y(t-\tau(t)))\left(T+\Pi^{T} T_{H}\right)^{T} y(t)+y^{T}(t)\left(T+\Pi^{T} T_{H}\right) F_{1}(y(t-\tau(t)))\right) \\
& \quad \leq e^{\alpha t} \beta\left(\left(\frac{1-\eta}{\beta}\right)^{-1} y^{T}(t) W_{\Pi} W_{\Pi}^{T} y(t)+\frac{1-\eta}{\beta} F_{1}^{T}(y(t-\tau(t))) F_{1}(y(t-\tau(t)))\right) \\
& \quad \leq e^{\alpha t}\left(\frac{\beta^{2}}{1-\eta} y^{T}(t) W_{\Pi} W_{\Pi}^{T} y(t)+(1-\eta)\left(y^{T}(t-\tau(t)) Q y(t-\tau(t))\right)\right) \\
& \quad \leq e^{\alpha t}\left(\frac{\beta^{2}}{1-\eta} y^{T}(t) \bar{W}_{\Pi} \bar{W}_{\Pi}^{T} y(t)+(1-\eta)\left(y^{T}(t-\tau(t)) Q y(t-\tau(t))\right)\right) .
\end{aligned}
$$


In view of $Q>0, \dot{\tau}(t) \leq \eta<1,(3.8)-(3.11)$, and $\left(H_{3}\right)$, it follows from Lemma 2.2 that

$$
\begin{aligned}
D^{+} V(y(t)) \leq & e^{\alpha t} y^{T}(t)\left(\beta(\alpha I-2 A)+e^{\alpha \tau_{0}} Q\right. \\
& \left.+\beta^{2}\left(\bar{W} \bar{W}^{T}+\frac{1}{1-\eta} \bar{W}_{\Pi} \bar{W}_{\Pi}^{T}\right)+L_{0}^{T} L_{0}\right) y(t) \leq 0 .
\end{aligned}
$$

From (3.12), we have

$$
V(y(t)) \leq V\left(y\left(t_{0}\right)\right)
$$

From (3.9) and (3.13), we can know that the solution $y(t)$ to system (3.2) satisfies

$$
\begin{aligned}
e^{\alpha t} \beta\|y(t)\|^{2} & =e^{\alpha t}\left(\beta y^{T}(t) y(t)\right) \leq V(y(t)) \leq V\left(y\left(t_{0}\right)\right) \\
& \leq\left(\beta+\frac{1}{\alpha}\left(e^{\alpha \tau_{0}}-1\right) \lambda_{\max }(Q)\right) \sup _{-\tau_{0} \leq \theta \leq 0}\|\varphi(\theta)\| .
\end{aligned}
$$

So,

$$
\left\|u(t, \xi)-u^{*}\right\|^{2} \leq J e^{-\alpha t} \sup _{-\tau_{0} \leq \theta \leq 0}\left\|\xi(\theta)-u^{*}\right\|^{2}
$$

where $J=1+1 / \alpha \beta\left(e^{\alpha \tau_{0}}-1\right) \lambda_{\max }(Q)$, for any $\xi(\theta) \in C\left(\left[-\tau_{0}, 0\right], R^{n}\right)$.

If there exists another equilibrium $u^{* *}=\left[u_{1}^{* *}, u_{2}^{* *}, \ldots, u_{n}^{* *}\right]^{T}$ of system $(2.1)$, we have $\left|u_{i}^{*}-u_{i}^{* *}\right| \leq\left|u_{i}(t, \xi)-u_{i}^{*}\right|+\left|u_{i}(t, \xi)-u_{i}^{* *}\right| \rightarrow 0, t \rightarrow \infty, i=1, \ldots, n$.

From the above proof, the system (2.1) has a unique equilibrium point $u^{*}$, which is globally exponentially robustly stable. Theorem 3.1 is proved.

Remark 3.2. When $a_{i}=\underline{a}_{i}>0, \underline{b}_{i j}=b_{i j}=\bar{b}_{i j}, \underline{w}_{i j}=w_{i j}=\bar{w}_{i j}, \underline{w}_{i j l}^{(k)}=w_{i j l}^{(k)}=\bar{w}_{i j l}^{(k)}, \underline{V}_{i}=V_{i}=\bar{V}_{i}$, the system (2.1) becomes to system in $[12,14]$. So, the system in $[12,14]$ is special case of system (2.1).

\section{Conclusion}

We have investigated global exponential robust stability of high-order hopfield neural networks with time-varying delays. By employing a new Lyapunov-Krasovskii functional and linear matrix inequality, some criteria of global exponential robust stability for the neural networks are established, which are easily verifiable.The systems found in the literature are special cases of the system (2.1). So the problem addressed in this paper should be a focused topic of theoretical as well as practical importance.

\section{Acknowledgments}

The authors would like to thank the anonymous reviewers for their valuable comments and suggestions. This research was fully supported by the National Natural Science Foundation of China (no. 11171374) and the Natural Science Foundation of Shandong (no. ZR2011AZ001) 


\section{References}

[1] X. Liao and Y. Liao, "Stability of Hopfield-type neural networks. II," Science in China. Series A. Mathematics, vol. 40, no. 8, pp. 813-816, 1997.

[2] G. Wallis, "Stability criteria for unsupervised temporal association networks," IEEE Transactions on Neural Networks, vol. 16, no. 2, pp. 301-311, 2005.

[3] C. Wang and D. J. Hill, "Deterministic learning and rapid dynamical pattern recognition," IEEE Transactions on Neural Networks, vol. 18, no. 3, pp. 617-630, 2007.

[4] J. Cao and M. Xiao, "Stability and Hopf bifurcation in a simplified BAM neural network with two time delays," IEEE Transactions on Neural Networks, vol. 18, no. 2, pp. 416-430, 2007.

[5] K. Gopalsamy and X. Z. He, "Stability in asymmetric Hopfield nets with transmission delays," Physica D. Nonlinear Phenomena, vol. 76, no. 4, pp. 344-358, 1994.

[6] L. Wang, "Comments on "robust stability for interval Hopfield neural networks with time delay" by X. F. Liao," IEEE Transactions on Neural Networks, vol. 13, no. 1, pp. 250-251, 2002.

[7] L. S. Wang, Delayed Recurrent Neural Networks, Science Press, Beijing, China, 2008.

[8] D. Xu, H. Zhao, and H. Zhu, "Global dynamics of Hopfield neural networks involving variable delays," Computers \& Mathematics with Applications, vol. 42, no. 1-2, pp. 39-45, 2001.

[9] P. P. Civalleri, M. Gilli, and L. Pandolfi, "On stability of cellular neural networks with delay," IEEE Transactions on Circuits and Systems. I. Fundamental Theory and Applications, vol. 40, no. 3, pp. 157-165, 1993.

[10] J. J. Hopfield, "Neural networks and physical systems with emergent collective computational abilities," Proceedings of the National Academy of Sciences of the United States of America, vol. 79, no. 8, pp. 2554-2558, 1982.

[11] J. J. Hopfield, "Neurons with graded response have collective computational like those of two-state neurons," Proceedings of the National Academy of Sciences of the United States of America, vol. 81, pp. 3088-3092, 1984.

[12] X. Liu and Q. Wang, "Impulsive stabilization of high-order Hopfield-type neural networks with timevarying delays," IEEE Transactions on Neural Networks, vol. 19, no. 1, pp. 71-79, 2008.

[13] Y. Wang, C. Lu, G. Ji, and L. Wang, "Global exponential stability of high-order Hopfield-type neural networks with S-type distributed time delays," Communications in Nonlinear Science and Numerical Simulation, vol. 16, no. 8, pp. 3319-3325, 2011.

[14] B. Xu, X. Liu, and X. Liao, “Global asymptotic stability of high-order Hopfield type neural networks with time delays," Computers \& Mathematics with Applications, vol. 45, no. 10-11, pp. 1729-1737, 2003.

[15] M. Brucoli, L. Carnimeo, and G. Grassi, "Associative memory design using discrete-time secondorder neural networks with local interconnections," IEEE Transactions on Circuits and Systems I: Fundamental Theory and Applications, vol. 44, no. 2, pp. 153-158, 1997.

[16] E. B. Kosmatopoulos and M. A. Christodoulou, "Structural properties of gradient recurrent highorder neural networks," IEEE Transactions on Circuits and Systems II: Analog and Digital Signal Processing, vol. 42, no. 9, pp. 592-603, 1995.

[17] E. B. Kosmatopoulos, M. M. Polycarpou, M. A. Christodoulou, and P. A. Ioannou, “High-order neural network structures for identification of dynamical systems," IEEE Transactions on Neural Networks, vol. 6, no. 2, pp. 422-431, 1995.

[18] A. Dembo, O. Farotimi, and T. Kailath, "High-order absolutely stable neural networks," IEEE Transactions on Circuits and Systems, vol. 38, no. 1, pp. 57-65, 1991.

[19] Y. Wang, P. Lin, and L. Wang, "Exponential stability of reaction-diffusion high-order Markovian jump Hopfield neural networks with time-varying delays," Nonlinear Analysis: Real World Applications, vol. 13, no. 3, pp. 1353-1361, 2012.

[20] S. Boyd, L. El Ghaoui, E. Feron, and V. Balakrishnan, Linear Matrix Inequalities in System and Control Theory, vol. 15 of SIAM Studies in Applied Mathematics, SIAM, Philadelphia, Pa, USA, 1994.

[21] S. G. Hu, Nonlinear Analysis and Methods, Huazhong University of Science and Technology, Wuhan, China, 1993.

[22] D. J. Guo, J. X. Sun, and Z. L. Liu, Functional Methods of Nonlinear Ordinary Differential Equations, Shandong Science Press, Jinan, China, 1995.

[23] L. Wang, R. Zhang, and Y. Wang, "Global exponential stability of reaction-diffusion cellular neural networks with S-type distributed time delays," Nonlinear Analysis. Real World Applications, vol. 10, no. 2, pp. 1101-1113, 2009. 


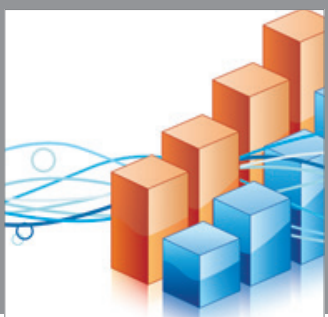

Advances in

Operations Research

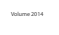

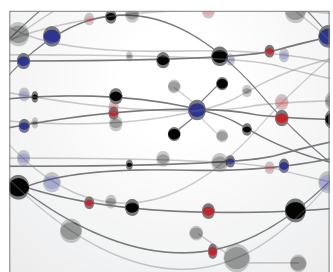

\section{The Scientific} World Journal
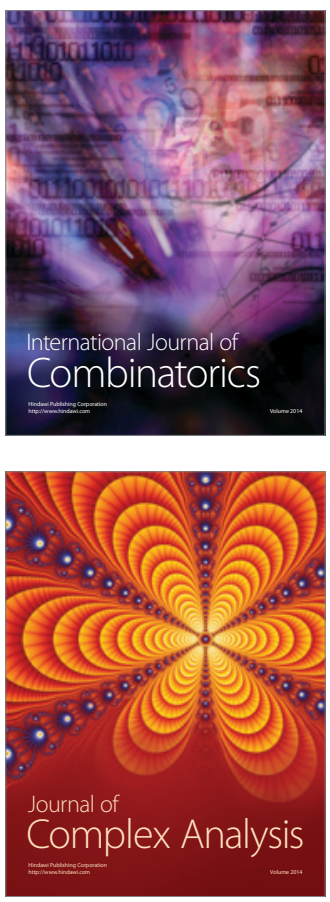

International Journal of

Mathematics and

Mathematical

Sciences
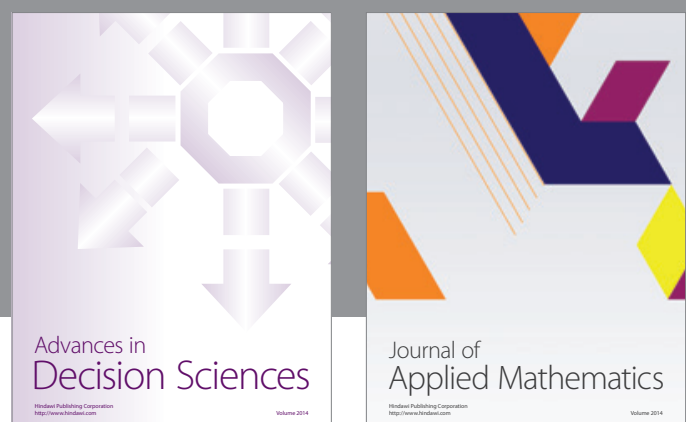

Journal of

Applied Mathematics
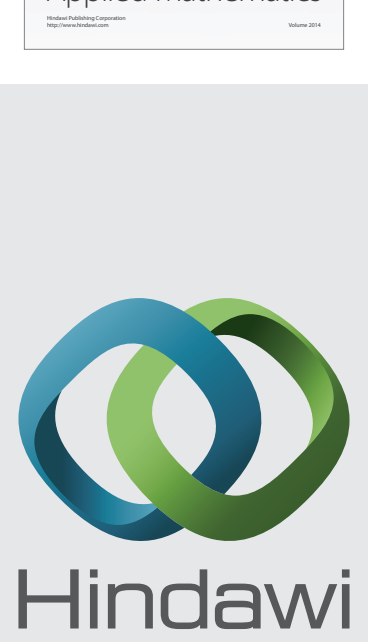

Submit your manuscripts at http://www.hindawi.com
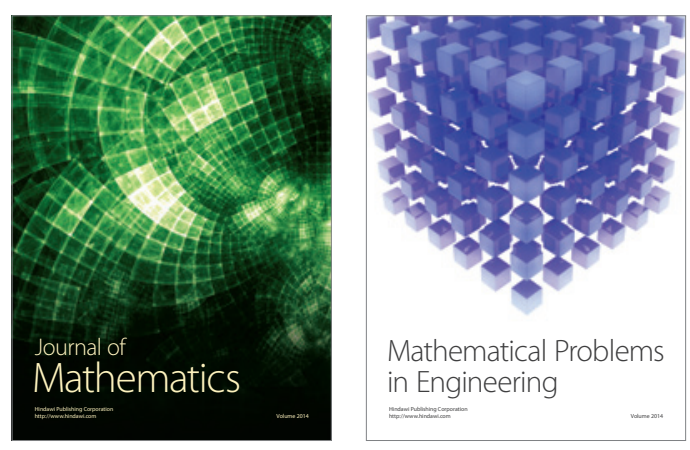

Mathematical Problems in Engineering
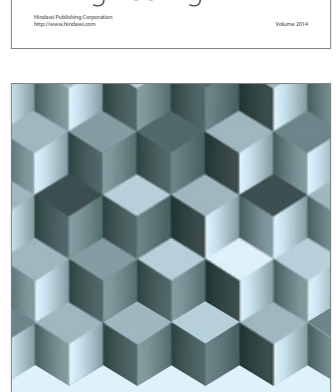

Journal of

Function Spaces
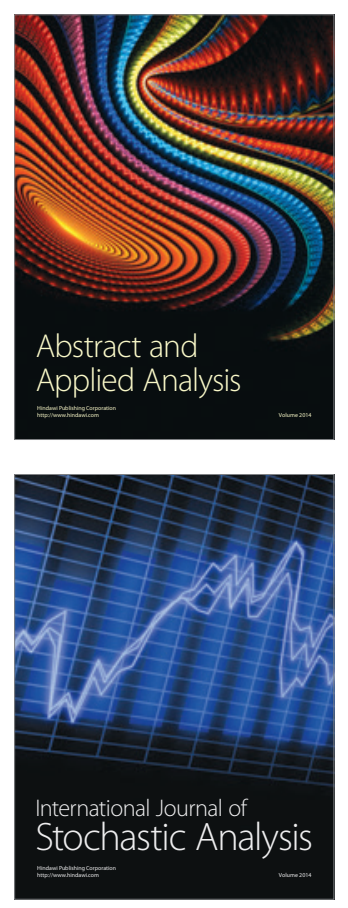

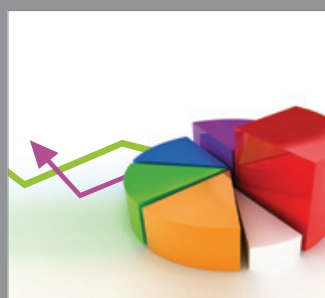

ournal of

Probability and Statistics

Promensencen
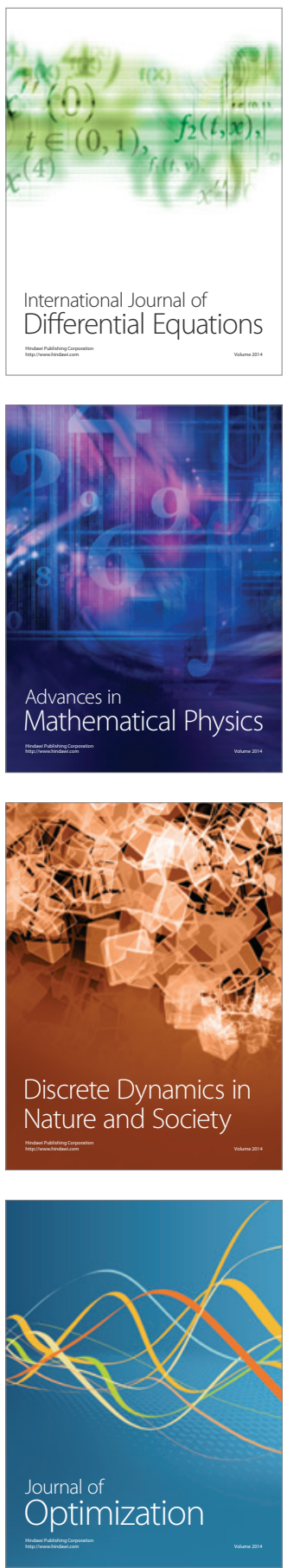